\title{
Conditions for patchiness in plankton models
}

Fabio Della Rossa

Stefano Fasani

Sergio Rinaldi (rinaldi@iiasa.ac.at)

\section{Approved by}

Ulf Dieckmann

Director, Evolution and Ecology Program

February 2015 Institute, its National Member Organizations, or other organizations supporting the work. 


\title{
Conditions for patchiness in plankton models
}

\author{
Fabio Della Rossa ${ }^{a}$, Stefano Fasani ${ }^{a}$, Sergio Rinaldi ${ }^{a, b}$ \\ ${ }^{a}$ DEI, Politecnico di Milano, Via Ponzio 34/5, 20133 Milano, Italy \\ ${ }^{b}$ Evolution and Ecology Program, International Institute for Applied Systems Analysis \\ 2361 Laxenburg, Austria
}

\begin{abstract}
Plankton patchiness in homogeneous physical environments is studied in this paper assuming that all involved populations disperse diffusively. A recent but powerful sufficient condition for the emergence of spatial patterns in models with any number of species is systematically applied to all food chain and food web plankton models and the result is rather sharp: All models explicitly containing phytoplankton, zooplankton and planktivorous fish suggest zooplankton patchiness, while models not containing phytoplankton or fish populations do not. The results are in agreement with many previous but particular theoretical studies on plankton patchiness and Turing instability, and testable prediction of the models satisfying the sufficient predictions is that zooplankton should be more patchy then phytoplankton, a property that is often seen in natural settings. An application to a complex model with five compartments (nutrient, phytoplankton, zooplankton, planktivorous fish, carnivorous fish) highlights the predictive power of the method.
\end{abstract} Keywords: plankton patchiness, plankton models, diffusive instability, Turing instability, dispersal, spatial pattern

Email address: sergio.rinaldi@polimi.it (Sergio Rinaldi) 


\section{Introduction}

Plant and animal populations are often not uniformly distributed in space, in particular when turbulent flows are controlling their dispersal. In aquatic ecosystems, the paradigmatic example is plankton patchiness that has attracted the attention of many researchers since the very beginning of spatial ecology (Levin and Segel, 1976, Steele, 1978, Okubo, 1992). In general, when populations interact demographically in media that vary in time and space one is, at least in principle, forced to study the problem through the use of two distinct submodels connected in cascade as sketched in Fig. 1. This makes the problem rather complex, because population samples not only contain information on demography but also on the characteristics of the physical environment, at the point of being sometimes dominated by them. For example, all techniques used for extracting the Liapunov exponent (the most popular indicator of chaos) from a plankton time series (Sugihara and May, 1990, Ascioti, Beltrami, Carroll and Wirick, 1993, Pascual, Ascioti and Caswell, 1995) would give an estimate (actually an upper bound) of the Liapunov exponent of the environmental submodel (Rinaldi and Solidoro, 1998, Colombo, Dercole and Rinaldi, 2008). That is to say, the plankton community plays the role of an instrument that measures a characteristic parameter of the environment, as noted by Pascual et al. (1995), who discovered that the Liapunov exponent extracted from plankton time series was actually very close to the Liapunov exponent emerging from the analysis of purely oceanographic time series.

Only a few studies have been carried out on complete models of the 


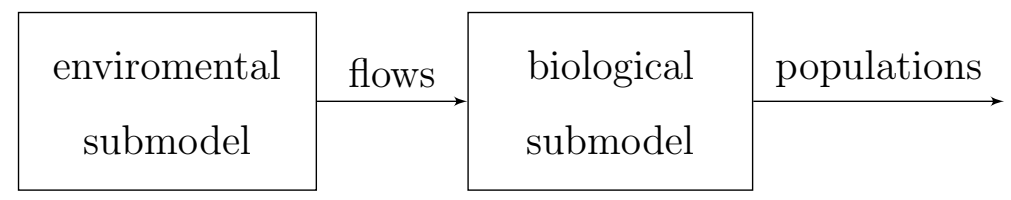

Figure 1: Two submodels connected in cascade.

kind shown in Fig.1 (Vilar, Solé and Rubí, 2003, Abraham, 1998, Hillary and Bees, 2004b, Colombo et al., 2008). These studies confirm that the characteristics of the environment can be dominant, but that in some cases plankton demography may add extra complexity.

In order to simplify the study of plankton, one can restrict the analysis to only one of the two submodels in Fig. 1. The first extreme approach consists of studying the spatio-temporal dynamics of the flows and deduce from them the population patterns by considering plankton, as well as other species, as inert particles (see Gower (1980) for an early support of this idea). In this way, the problem is reduced to a relatively standard problem of hydrodynamics where only the sinking, floating or swimming characteristics of the populations are taken into account. This approach can explain numerous plankton patterns, observed at various spatial scales, like vortices that turn on and off alternatively (Aref, 1984), multiple bands of dense organisms lumped into swaths (Shanks, 1983), and long single stripes of swimming or floating plankton parallel to shore (Franks, 1997). Conversely, the second extreme approach, simply rules out the hydrodynamics by assuming that all flows are constant in time and space, so that the model becomes a classical population model with a dispersal mechanism controlling the movement of the individuals in a spatial domain. This approach has a long scientific tra- 
dition and emphasizes the role of biology by pointing out that demography has the power of creating surprisingly complex spatial patterns, like spots and stripes of abundances, even in perfectly homogeneous environments.

Segel and Jackson (1972) were the first to show that the theory developed by Turing (1952) in his celebrated study on the origin of morphogenesis could be applied in ecology to check if density dependent mechanisms could promote spatial pattern formation in perfectly homogeneous environments. Their study was limited to models with two populations and diffusive dispersal, because these were Turing's assumptions. A few years later, Levin and Segel (1976) conjectured that Turing's theory was potentially the most appropriate tool for supporting the idea that plankton patchiness could be the consequence of demographic characteristics of the populations. However, the phytoplankton-zooplankton model they used is not credible (phytoplankton in the absence of zooplankton increases unboundedly) and suggests that the so-called "activator" (a key notion in Turing's theory) is phytoplankton. This implies that phytoplankton should be more patchy than zooplankton, a property which is in contrast with observations (Levin, 1992, Vilar et al., 2003). Here we show that Levin and Segel could not do any better, given the constraint of using a model with only two populations. In fact, all standard ditrophic food chain models with zooplankton at the top or at the bottom of the chain can not have zooplankton as activator (we consider as non-standard not only the models with unboundedly growing phytoplankton (Levin and Segel, 1976), but also those with predator with ratio-dependent functional responses which are known to be prone to degeneracies (Yodzis, 1994, Abrams, 1994). By contrast Levin and Segel were right in making 
their conjecture because, as shown in this paper, a recently derived sufficient condition for pattern formation (Satnoianu, Menzinger and Maini, 2000, Satnoianu and van den Driessche, 2005, Della Rossa, Fasani and Rinaldi, 2012), is structurally satisfied in all realistic models where zooplankton feeds on phytoplankton and is predated by fish.

The paper is organized as follows. In the next section we briefly report the sufficient condition implying pattern formation in models with more than two populations. Then, we show that under very general assumptions such a condition is satisfied in models with (one or more groups of) phytoplankton, zooplankton and planktivorous fish. This is done by distinguishing between zooplankton and phytoplankton patchiness. Finally, we highlight the power of our condition by simulating a model with five compartments, namely nutrient, phytoplankton, zooplankton, planktivorous fish, and carnivores. A final section presents the conclusions and discusses possible extensions.

\section{A simple sufficient condition for pattern formation}

Assume that $n$ populations with densities $x_{i}, i=1, \ldots, n$ depending upon time and space interact in a spatial domain in accordance with a standard reaction-diffusion PDE

$$
\frac{\partial x_{i}}{\partial t}=f_{i}(x)+d_{i} \nabla^{2} x_{i} \quad i=1, \ldots, n
$$

where $d_{i}$ is dispersal of $i$-th population. In general, zero-flux or periodic conditions are imposed at the boundary of the spatial domain. If $d_{i}$ and $f_{i}$ do not depend on time and space, then a uniform solution $\bar{x}=\left(\bar{x}_{1}, \ldots, \bar{x}_{n}\right)$ of 
91

(1) (satisfying $\partial x_{i} / \partial t=\nabla^{2} x_{i}=0$ ) is an equilibrium of the lumped model

$$
\frac{d x_{i}}{d t}=f_{i}(x) \quad i=1, \ldots, n
$$

2

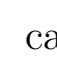
anced dispersal rates. This somehow counterintuitive phenomenon, called diffusive (or Turing) instability, has been extensively used in ecology in the last 40 years to discuss the problem of pattern formation in spatially extended ecosystems (see, for instance, Segel and Jackson (1972), Levin and Segel (1976), Chakraborty, Singh, Lucy and Ridland (1996), Bartumeus, Alonso and Catalan (2001), Alonso, Bartumeus and Catalan (2002), Baurmann, Gross and Feudel (2007), Wang, Liu and Jin (2007), Zhang, Wang and Xue (2009), Sun, Zhang and Jin (2009), Banerjee (2010), Fasani and Rinaldi (2011, 2012), Della Rossa et al. (2012)). Also the problem of plankton patchiness has been studied in terms of diffusive instability (Levin and Segel, 1976, Malchow, 1993, 1994), even if it has more often been dealt with through the analysis of power spectra (see, for example, Steele and Henderson (1992), Powell and Okubo (1994), Abraham (1998), Vilar et al. (2003)).

Necessary and sufficient conditions for diffusive instability have first been obtained by Turing for the particular case $n=2$ and then by Satnoianu et al. (2000), Satnoianu and van den Driessche (2005) for the general case. In this paper, we only use the following sufficient condition for diffusive instability that can be easily derived (see Della Rossa et al. (2012)) from the general results of Satnoianu and coauthors.

A sufficient condition for diffusive instability. If a population, say the $i$-th one, is an activator, in the sense that $\partial f_{i} / \partial x_{i}>0$ at a positive stable 
equilibrium $\bar{x}$ of (2), then the uniform solution $\bar{x}$ of (1) is unstable provided the activator disperses sufficiently less than the other populations. The existence of an activator is only a sufficient condition for diffusive instability, in the sense that there are systems with three or more species in which diffusive instability can emerge even if there are no activators. In contrast, this is not possible in systems with only two species, where the existence of an activator is a necessary and sufficient condition of diffusive instability, again under the assumption of unbalanced dispersals.

In the case $n=2$, there can be only one activator because, $\bar{x}$ being stable, the trace of the Jacobian $\left(\partial f_{1} / \partial x_{1}+\partial f_{2} / \partial x_{2}\right)$ must be negative. By constrast, when $n>2$, we can have multiple activators, in which case the dispersals of the activators required to guarantee spatial patterns do not need to be unbalanced.

The spatial patterns that emerge when the sufficient condition is satisfied (typically spot-like patterns) are particularly sharp for the activators and depend upon demographic parameters and dispersal, as shown in the application described in Sect.4.

\section{Patchiness in plankton models}

Phytoplankton $(P)$ and zooplankton $(Z)$ populations are central components of the aquatic food web going from nutrient $(N)$ to fish $(F)$. They are usually present in a high number of groups characterized by different size, mobility and life strategies. Each phytoplankton group is limited by light and nutrients (typically, nitrogen and phosphorous) and is predated by a few zooplankton groups, which, in turn, are the food sources of a number 
of fish species. Some demographic characteristics of the various components of the food web are influenced by periodic (or almost periodic) exogenous factors (daily cycle for light, weekly cycle for nutrient production, moon cycle for predator efficiency, yearly cycle for light and water temperature) that can have relevant impacts on plankton dynamics (see, for example, May (1974), Harris (1986), Berryman and Millstein (1989), Sugihara and May (1990), Scheffer (1991b), Steele and Henderson (1992), Hastings, Hom, Ellner, Turchin and Godfray (1993), Ascioti et al. (1993), Pascual et al. (1995)). Depth is also an important independent variable that, in principle, should be included in any model in order to carefully describe the impact of self-shading on phytoplankton growth.

Models used to mimic spatio-temporal plankton dynamics are much more simple than reality. In particular, in order to apply our sufficient condition for diffusive instability we rule out exogenous periodicities and depth. Thus, the models we will consider are, in the most complex case, food webs with constant demographic parameters and dispersal. But, more often, they simply mimic food chains going from nutrients to fish or segments of this food chain.

The aim of our analysis is to show that, under general and standard assumptions, zooplankton populations are activators, in the sense specified in the previous section. This occurs with almost no exception if the model includes explicitly (as it should!) phytoplankton and fish. By contrast, if preys or enemies of zooplankton are missing, then there is no chance that the model predicts zooplankton patchiness. In order to support these statements, we first show that in food chain models ending with zooplankton 
(e.g. phytoplankton-zooplankton $(P-Z)$ models or nutrient-phytoplanktonzooplankton $(N-P-Z)$ models), or starting with zooplankton (e.g. zooplanktonfish $(Z-F)$ models), zooplankton can not be an activator. Then, we prove that food chain models including prey and enemies of zooplankton, like $N-P-Z-F$ models, satisfy our sufficient condition for the emergence of zooplankton patchiness. Finally, we show that the result remains valid also in food webs, i.e., when the model includes multiple groups of phytoplankton and/or zooplankton.

\subsection{Zooplankton patchiness}

Food chain models with zooplankton at the top of the chain are either $P-Z$ models (Levin and Segel, 1976, Steele and Henderson, 1992, Vilar et al., 2003) or $N-P-Z$ models (Steele and Henderson, 1992, Abraham, 1998, Hillary and Bees, 2004a,b). They all share the same equation for zooplankton, namely

$$
\begin{aligned}
\frac{d Z}{d t}=f_{Z}(P, Z) & =e_{Z} \Psi_{Z}(P, Z) Z-m_{Z}(Z) Z \\
& =Z\left[e_{Z} \Psi_{Z}(P, Z)-m_{Z}(Z)\right]
\end{aligned}
$$

where $e_{Z}, m_{Z}$ and $\Psi_{Z}$ are efficiency, per-capita mortality and functional response of zooplankton. If we assume, that the stable equilibrium $(\bar{N}, \bar{P}, \bar{Z})$ is strictly positive (zooplankton patchiness in the absence of zooplankton makes no sense) then

$$
\frac{\partial f_{Z}}{\partial Z}=\bar{Z}\left[e_{Z} \frac{\partial \Psi_{Z}}{\partial Z}-\frac{\partial m_{Z}}{\partial Z}\right]
$$

because the term in the brackets in (3) is zero at a positive equilibrium. If the functional response $\Psi_{Z}$ and the per-capita mortality $m_{Z}$ of zooplankton 
do not depend on zooplankton density, then $\partial f_{Z} / \partial Z=0$ so that zooplankton is not an activator (even if our sufficient condition is 'almost satisfied'). If zooplankton individuals interfere when predating $\left(\partial \Psi_{Z} / \partial Z<0\right)$ and/or suffer some kind of intraspecific competition $\left(\partial m_{Z} / \partial Z>0\right)$, then $\partial f_{Z} / \partial Z$ is negative and the sufficient condition for pattern formation is not satisfied. In order to obtain the opposite result, one should imagine that zooplankton is cooperative in searching for food $\left(\partial \Psi_{Z} / \partial Z>0\right)$ or in activating survival mechanisms $\left(\partial m_{Z} / \partial Z<0\right)$, but these assumptions are not sensible and in fact they have never been reported in the literature.

Food chain models with zooplankton at the bottom of the chain (never discussed in the literature so far) should be $Z-F$ models with the fish equation of the form

$$
\frac{d F}{d t}=f_{F}(Z, F)=e_{F} \Psi_{F}(Z, F) F-m_{F}(F) F=F\left[e_{F} \Psi_{F}(Z, F)-m_{F}(F)\right]
$$

so that, at a positive equilibrium $(\bar{Z}, \bar{F})$,

$$
\frac{\partial f_{F}}{\partial F}=\bar{F}\left[e_{F} \frac{\partial \Psi_{F}}{\partial F}-\frac{\partial m_{F}}{\partial F}\right]
$$

The standard assumption in studies of fish stocks (Walters and Martell, 2004) is that the functional response $\Psi_{F}$ and the per-capita mortality $m_{F}$ do not depend on $F$ (notice that this rules out the case of ratio-dependency), so that from (5) $\partial f_{F} / \partial F=0$. Since, by assumption, the equilibrium $(\bar{Z}, \bar{F})$ is stable, the trace of the Jacobian $\left(\partial f_{Z} / \partial Z+\partial f_{F} / \partial F\right)$ must be negative, and hence $\partial f_{Z} / \partial Z<0$, i.e. our sufficient condition is not satisfied for zooplankton. This conclusion is reinforced if $\partial \Psi_{F} / \partial F>0$ and/or $\partial m_{F} / \partial F<0$, i.e. if fish are cooperative and/or predated by Holling type II carnivores. Thus, in 
conclusion, even $Z-F$ models do not suggest zooplankton patchiness under very general and realistic assumptions.

Let us now consider $P-Z-F$ and $N-P-Z-F$ models where both prey and predator of zooplankton appear explicitly. Models of this kind have been proposed in the literature with $N$ and $F$ fixed at constant values and used as control parameters (in order to still obtain a model with only two differential equations) (Scheffer, 1991b,a, Malchow, 1993, 1994, Medvinsky, Petrovskii, Tikhonova, Malchow and Li, 2002) or with $N$ and $F$ varying in time in accordance with a differential equation (Doveri, Scheffer, Rinaldi, Muratori and Kuznetsov, 1993, Rinaldi and Solidoro, 1998). In all these cases, the zooplankton equation is still eq. (3) but with the addition of an extra mortality due to fish, namely

$$
\begin{aligned}
\frac{d Z}{d t}=f_{Z}(P, Z, F) & =e_{Z} \Psi_{Z}(P, Z) Z-m_{Z}(Z) Z-F \Psi_{F}(Z, F) \\
& =Z\left[e_{Z} \Psi_{Z}(P, Z)-m_{Z}(Z)-F \frac{\Psi_{F}(Z, F)}{Z}\right]
\end{aligned}
$$

In the absence of interference and cooperation in the zooplankton population $\left(\partial \Psi_{Z} / \partial Z=\partial m_{Z} / \partial Z=0\right)$ we obtain from (6) that at a positive equilibrium $\bar{Z}$

$$
\frac{\partial f_{Z}}{\partial Z}=-\bar{Z} \bar{F} \frac{\partial\left(\Psi_{F} / Z\right)}{\partial Z}
$$

To evaluate the sign of $\partial f_{Z} / \partial Z$ in (7) we can consider the two standard cases of fish functional response

$$
\Psi_{F}= \begin{cases}a Z /(b+Z) & \text { Holling type II } \\ a Z^{2} /\left(b^{2}+Z^{2}\right) & \text { Holling type III }\end{cases}
$$

where $b$ is the half-saturation constant, namely the density of zooplankton 
at which fish predation is half maximum. After some algebra, we obtain

$$
\frac{\partial f_{Z}}{\partial Z}=\left\{\begin{array}{l}
a \bar{Z} /(b+\bar{Z})^{2} \\
a \bar{Z}\left(\bar{Z}^{2}-b^{2}\right) /\left(b^{2}+\bar{Z}^{2}\right)^{2}
\end{array}\right.
$$

and the conclusion is that if the fish has a Holling type II functional response the zooplankton is always an activator, while in the case of Holling type III functional response the zooplankton is an activator if $\bar{Z}>b$, i.e. if the equilibrium $(\bar{N}, \bar{P}, \bar{Z}, \bar{F})$ is a so called zooplankton dominated equilibrium (Malchow, 1993). In the opposite case, namely when the equilibrium is a phytoplankton dominated equilibrium (i.e. $\bar{P}$ large and $\bar{Z}$ small) zooplankton is not an activator and its patchiness can not be inferred from our sufficient condition. This is not a great deal because knowing if a population with low abundance is patchy or not is only a futile curiosity.

The results obtained so far are valid also in food web models characterized by multiple phytoplankton and zooplankton groups (see, for example, Rose, Swartzman, Kindig and Taub (1988)). In these models each zooplankton group $i$ is described by an equation similar to (6)

$$
\frac{d Z^{(i)}}{d t}=e_{Z}^{(i)} \Psi_{Z}^{(i)} Z^{(i)}-m_{Z}^{(i)}(Z) Z^{(i)}-F \Psi_{F}^{(i)}
$$

where the $i$-th functional response $\Psi_{Z}^{(i)}$ depends upon the phytoplankton groups that are in the diet of the $i$-th zooplankton group but not upon $Z^{(i)}$, while the fish functional response $\Psi_{F}^{(i)}$ depends upon $Z^{(i)}$ but also upon the other zooplankton groups. More precisely, $\Psi_{F}^{(i)}$ takes the form

$$
\Psi_{F}^{(i)}=\frac{a Z^{(i)}}{b_{0}+b_{1} Z^{(1)}+b_{2} Z^{(2)}+\ldots+b_{i} Z^{(i)}+\ldots}
$$

in the case of a generalized type II fish functional response, or a similar form in the case of a generalized type III fish functional response. Substituting 
243

244

(9) into (8) one obtains

$$
\frac{d Z^{(i)}}{d t}=Z^{(i)}\left[e_{Z}^{(i)} \Psi_{Z}^{(i)}-m_{Z}^{(i)}-\frac{a F}{b_{0}+b_{1} Z^{(1)}+b_{2} Z^{(2)}+\ldots+b_{i} Z^{(i)}+\ldots}\right]
$$

that gives

$$
\frac{\partial f_{Z}^{(i)}}{\partial Z^{(i)}}=\frac{a F b_{i} \bar{Z}^{(i)}}{\left(b_{0}+b_{1} Z^{(1)}+b_{2} Z^{(2)}+\ldots+b_{i} Z^{(i)}+\ldots\right)^{2}}
$$

which is always positive, thus indicating that each zooplankton group is an activator. As in the case of food chain models, the analysis of model $(8,9)$ with type III fish functional response brings to the conclusion that zooplankton groups are activators in zooplankton dominated regimes.However, not all zooplankton groups have the same chance to be patchy, because some of them might not satisfy the condition of sufficiently low dispersal. This result might be of some interest for interpreting the dependence of patchiness upon individual size recently pointed out in a study on field data (Decima, Ohman and De Robertis, 2010).

\subsection{Phytoplankton patchiness}

The analysis performed for zooplankton can be repeated to check if also phytoplankton is an activator in $N-P-Z$ or $N-P-Z-F$ models. In these models the phytoplankton equation is

$$
\begin{aligned}
\frac{d P}{d t}=f_{P}(N, P, Z) & =e_{P} \Psi_{P}(P, N) P-m_{P}(P) P-Z \Psi_{Z}(P, Z) \\
& \left.=P\left[e_{P} \Psi_{P}(P, N)-m_{P}(P)-Z \frac{\Psi_{Z}(P, Z)}{P}\right)\right]
\end{aligned}
$$

where $\Psi_{P}$ is nutrient uptake of phytoplankton. Thus,

$$
\frac{\partial f_{P}}{\partial P}=\bar{P}\left[e_{P} \frac{\partial \Psi_{P}}{\partial P}-\frac{\partial m_{P}}{\partial P}-Z \frac{\partial\left(\Psi_{Z} / P\right)}{\partial P}\right]
$$


and even in the simplest case of constant mortality $\left(\partial m_{P} / \partial P=0\right)$ and type II functional response of zooplankton $\left(\Psi_{Z}=a P /(b+P)\right)$ we can not obtain a unique answer. In fact

$$
\frac{\partial f_{P}}{\partial P}=\bar{P}\left[e_{P} \frac{\partial \Psi_{P}}{\partial P}+\frac{a \bar{Z}}{(b+\bar{P})^{2}}\right]
$$

and the first term in brackets is negative because self-shading is depressing the nutrient uptake of phytoplankton. Actually, eq. (11) shows that phytoplankton has higher chances to be an activator when its density is low and $\bar{Z}$ is high, i.e. in zooplankton dominated regimes. But this result. as shown in the next section, is not always guaranteed.

\subsection{Unbalance of dispersals}

We conclude this section with a comment on a somehow delicate point, namely that of the required unbalance of dispersals. Since zooplankton is in general an activator, its patchiness is guaranteed by our sufficient condition provided it disperses sufficiently less than phytoplankton and fish, i.e.

$$
d_{Z}<d_{P} \quad d_{Z}<d_{F}
$$

While there is no doubt on the latter condition, the first poses some problems. The most common opinion on this matter, in particular when phytoplankton and zooplankton are considered as inert traces, is that $d_{P}$ and $d_{Z}$ are roughly comparable (see, for instance, Medvinsky et al. (2002)). But the inequality $d_{Z}<d_{P}$ can, in principle, be supported by noticing that many zooplankton species are capable of resisting to currents by grasping, while phytoplankton is not. In reality, the mobility of zooplankton can be used to say that when there is no turbulence the opposite inequality can hold (see, for 
instance, Malchow (1994)). However, this issue is of no relevance when also phytoplankton is an activator, because in that case the only unbalance that matters is $d_{Z}<d_{F}$. From the above discussion it follows that this should often be the case in zooplankton dominated regimes.

\section{Analysis of a particular plankton model}

In this section we study in some detail a spatially extended food chain model with 5 components: Nutrient $(N)$, Phytoplankton $(P)$, Zooplankton $(Z)$, Planktivorous fish $(F)$, and Carnivores $(C)$. The zooplankton is the central compartment of the chain and we therefore expect zooplankton patchiness for suitably low zooplankton dispersal. The model, derived from Doveri et al. (1993), has been selected for three reasons: First, it has performed quite well in explaining the emergence of chaotic plankton dynamics in the presence of seasonalities (Rinaldi and Solidoro, 1998); second, realistic parameter ranges are available for it (Doveri et al., 1993); third it is much more detailed than the simple $P-Z$ or $P-Z-F$ models used until now for testing pattern formation due to Turing instability.

The model equations, obtained from Doveri et al. (1993) by eliminating 
all seasonalities, are:

$$
\begin{aligned}
\frac{d N}{d t}= & \delta\left(N_{0}-N\right)-\beta_{P} \frac{P N}{k_{P}+N} \frac{l}{\gamma P+l}+\rho_{P} m_{P} P+\rho_{Z} m_{Z} Z+\rho_{F} m_{F} F+ \\
& +\rho_{C} m_{C} C+\xi_{Z} \beta_{Z} \frac{Z P}{k_{Z}+P}+\xi_{F} \beta_{F} \frac{F Z}{k_{F}+Z} \\
\frac{d P}{d t}= & e_{P} \beta_{P} \frac{P N}{k_{P}+N} \frac{l}{\gamma P+l}-\beta_{Z} \frac{Z P}{k_{Z}+P}-m_{P} P-\delta P \\
\frac{d Z}{d t}= & e_{Z} \beta_{Z} \frac{Z P}{k_{Z}+P}-\beta_{F} \frac{F Z}{k_{F}+Z}-m_{Z} Z-\delta Z \\
\frac{d F}{d t}= & e_{F} \beta_{F} \frac{F Z}{k_{F}+Z}-\beta_{C} \frac{C F}{k_{C}+F}-m_{F} F-\delta F+V_{0} \bar{I} \\
\frac{d C}{d t}= & e_{C} \beta_{C} \frac{C F}{k_{C}+F}-m_{C} C
\end{aligned}
$$

Notice that the nutrient uptake per unit of phytoplankton decreases with phytoplankton density in order to take the effect of self-shading into account. Thus, phytoplankton is not guaranteed to be an activator.

The reference parameter values used in all simulations are reported in Table 1. They have been fixed in the feasible ranges proposed in Doveri et al. (1993) except the parameter $l$ which has been selected in order to enhance the self-shading effect.

For these reference parameter values the model has a stricly positive stable equilibrium

$$
\begin{aligned}
\bar{N} & =0.356 & & {\left[m g_{P} l^{-1}\right] } \\
\bar{P} & =0.075 & & {\left[m g_{d w} l^{-1}\right] } \\
\bar{Z} & =0.565 & & {\left[m g_{d w} l^{-1}\right] } \\
\bar{F} & =0.024 & & {\left[m g_{d w} l^{-1}\right] } \\
\bar{C} & =0.099 & & {\left[m g_{d w} l^{-1}\right] }
\end{aligned}
$$

which is a zooplankton dominated equilibrium. The Jacobian matrix evalu- 


\begin{tabular}{|c|c|c||c|c|c|}
\hline Parameter & Units & Value & Parameter & Units & Value \\
\hline$m_{P}$ & $d a y^{-1}$ & 0.2 & $m_{Z}$ & $d a y^{-1}$ & 0.15 \\
$m_{F}$ & $d a y^{-1}$ & 0.02 & $m_{C}$ & $d a y^{-1}$ & 0.01 \\
$k_{P}$ & $m g_{P} l^{-1}$ & 0.02 & $k_{Z}$ & $m g_{d w} l^{-1}$ & 0.075 \\
$k_{F}$ & $m g_{d w} l^{-1}$ & 0.5 & $k_{C}$ & $m g_{d w} l^{-1}$ & 1.2 \\
$\beta_{P}$ & $d a y^{-1}$ & 0.1 & $\beta_{Z}$ & $d a y^{-1}$ & 0.6 \\
$\beta_{F}$ & $d a y^{-1}$ & 0.2 & $\beta_{C}$ & $d a y^{-1}$ & 0.5 \\
$e_{P}$ & $m g_{d w}\left(m g_{P}\right)^{-1}$ & 100 & $e_{Z}$ & - & 0.6 \\
$e_{F}$ & - & 0.6 & $e_{C}$ & - & 1 \\
$\rho_{P}$ & $m g_{P}\left(m g_{d w}\right)^{-1}$ & 0.003 & $\rho_{Z}$ & $m g_{P}\left(m g_{d w}\right)^{-1}$ & 0.0031 \\
$\rho_{F}$ & $m g_{P}\left(m g_{d w}\right)^{-1}$ & 0.00465 & $\rho_{C}$ & $m g_{P}\left(m g_{d w}\right)^{-1}$ & 0.0054 \\
$\xi_{Z}$ & $m g_{P}\left(m g_{d w}\right)^{-1}$ & 0.007 & $\xi_{F}$ & $m g_{P}\left(m g_{d w}\right)^{-1}$ & 0.006 \\
$\delta$ & $d a y^{-1}$ & 0.025 & $l$ & $c a l m^{-2} d a y^{-1}$ & 4 \\
$\gamma$ & $c a l l m^{-2}\left(d a y m g_{d w}\right)^{-1}$ & 150 & $V_{0}$ & $m g_{d w} l^{-1}$ & 2 \\
$N_{0}$ & $m g_{P} l^{-1}$ & 0.37 & $\bar{I}$ & $d a y^{-1}$ & 0.00027 \\
\hline
\end{tabular}

Table 1: Parameter values for model (12)

313

ated numerically at this equilibrium is

$$
\left(\begin{array}{ccccc}
-0.0252777 & 0.00199401 & 0.00257325 & 0.000729839 & 0.000054 \\
0.0277658 & -0.70517 & -0.299329 & 0 & 0 \\
0 & 0.681537 & 0.00243975 & -0.10614 & 0 \\
0 & 0 & 0.00129449 & -0.0212353 & -0.01 \\
0 & 0 & 0 & 0.0399192 & 0
\end{array}\right)
$$

and the sign of its central element confirms that zooplankton is an activator.

The negativity of the second diagonal element shows, as observed in the previous section, that in a zooplankton dominated equilibrium there is no guarantee that phytoplankton is an activator.

Thus, on the basis of the results obtained with our sufficient condition 
we expect zooplankton patchiness if zooplankton dispersal is sufficiently low. To confirm this guess we have performed extensive simulations of the PDE model $(1,12)$ on $51.2 \mathrm{~km} \times 51.2 \mathrm{~km}$ square spatial domains with periodic boundary conditions. The initial conditions have been randomly selected, in order to avoid the formation of special patterns. Significant parts of the simulation results are reported in Fig. 2 (see captions for details) and confirm all our expected results. They point out that the zooplankton spatial patterns are typically spot-like patterns, where the spots become more numerous and irregular when the zooplankton dispersal decreases.
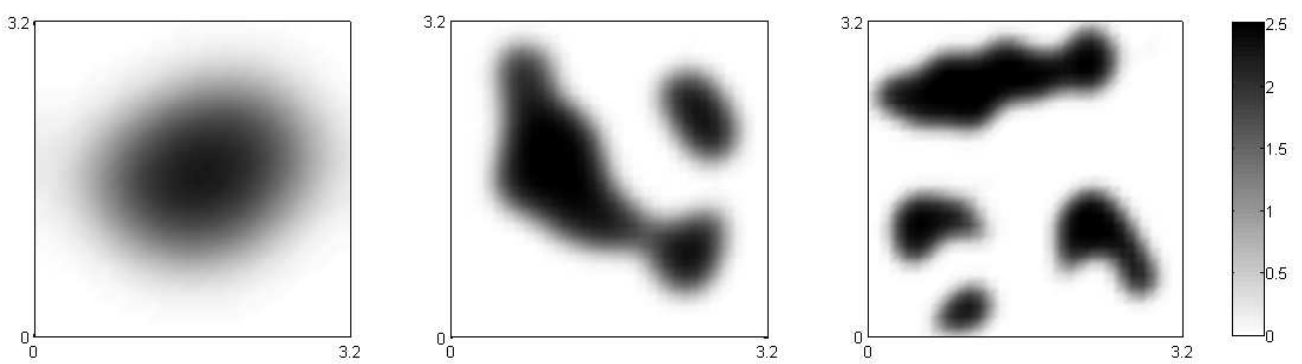

Figure 2: Stationary solutions of model $(1,12)$ obtained through simulations on $51.2 \mathrm{~km} \times$ $51.2 \mathrm{~km}$ square spatial domains with periodic boundary conditions and randomly selected initial conditions. In order to show more details on the shape and dimension of zooplankton spots, only parts of the solutions are shown, by zooming on $3.2 \mathrm{~km} \times 3.2 \mathrm{~km}$ squares. Parameter values are as in Table 1 and dispersal coefficients are $d_{N}=d_{P}=2 \mathrm{~km}^{2} \mathrm{day}^{-1}$, $d_{F}=d_{C}=10 \mathrm{~km}^{2} \mathrm{day}^{-1}$ and, from left to right, $d_{Z}=1,0.5,0.1 \mathrm{dm}^{2} \mathrm{day}^{-1}$. Simulations have been performed using GRIND for Matlab, http://www . aew.wur.nl/UK/GRIND/. 


\section{Concluding remarks}

We have studied in this paper the problem of plankton patchiness due to diffusive instability. For this we have used a very simple but powerful sufficient condition for pattern formation that can be applied when a diagonal element of the Jacobian matrix is positive. First we have systematically discussed all possible zooplankton models and discovered that the sufficient condition is satisfied if and only if the model contains both the prey (phytoplankton) and the predator (planktivorous fish) of zooplankton. The discovery that the impact of fish on zooplankton, a problem rarely considered in the technical literature, is the key for understanding pattern formation, gives a new value to old contributions in aquatic sciences (Rose, Swartzman, Kindig and Taub, 1975, Leah, Moss and Forrest, 1980, Cronberg, 1982, Shapiro and Wright, 1984, Reinertsen and Olsen, 1984, Levitan, Kerfoot and De Mott, 1985). Then, we have studied models involving phytoplankton and discovered that patchiness emerges if the effect of self-shading is not too strong and the equilibrium is zooplankton dominated. In conclusion, we have pointed out subtle but relevant differences between phytoplankton and zooplankton in the role they play in promoting patchiness.

The present study has been carried out under the assumption of diffusive dispersal of all populations, which is rigorously justified if all automotive individuals (like planktivorous fish and carnivores) undergo unbiased random walks. In contrast, if individuals of some species are also actively moving, it is, in principle, important to add to the diffusive term a second term (called cross-emigration) interpreting the tendencies of individuals to escape from predator and/or pursuit prey. In general this second dispersal mechanism 
is assumed to depend on the gradient of prey and predator abundances, (see, for example, Murray (1990), Arditi, Tyutyunov, Morgulis, Govorukhin and Senina (2001), Huang and Diekmann (2003), Li, Gao, Hui, Han and Shi (2005)) and the conclusion is that cross-emigration can either increase or decrease spatial complexity (Huang and Diekmann, 2003). It would therefore be interesting, though certainly not trivial, to apply Huang and Diekmann approach to the case in which planktivorous fish and carnivores have relevant cross-emigration responses.

Obviously, the present study could also be extended in other directions, for example by looking at the effect of depth, seasons and multiplicity of attractors. But certainly more attractive is the idea of checking if what we have discovered here can be extended up to the point of formulating a sort of general ecological principle, namely that populations that disperse less than their prey and enemies tend to be patchy under very general conditions.

\section{Acknowledgement}

The authors are grateful to Egbert van Nes (Wageningen University) for helpful advice and for providing his software "GRIND" for the simulation of spatial models. The Reviewers are also acknowledged for their numerous and constructive criticisms.

\section{References}

Abraham, E., 1998. The generation of plankton patchiness by turbulent stirring. Nature 391, 577-580. 
Abrams, P., 1994. The fallacies of ratio-dependent predation. Ecology 75, $1842-1850$.

Alonso, D., Bartumeus, F., Catalan, J., 2002. Mutual interference between predators can give rise to Turing spatial patterns. Ecology 83, 28-34.

Arditi, R., Tyutyunov, Y., Morgulis, A., Govorukhin, V., Senina, I., 2001. Directed movement of predators and the emergence of density-dependence in predator-prey models. Theoretical Population Biology 59, 207-221.

Aref, H., 1984. Stirring by chaotic advection. Journal of Fluid Mechanics $143,1-21$.

Ascioti, F., Beltrami, E., Carroll, T., Wirick, C., 1993. Is there chaos in plankton dynamics? J. Plankton Res. 15, 603-617.

Banerjee, M., 2010. Self-replication of spatial patterns in a ratio-dependent predator-prey model. Mathematical and Computer Modelling 51, $44-52$.

Bartumeus, F., Alonso, D., Catalan, J., 2001. Self-organized spatial structures in a ratio-dependent predator-prey model. Physica A: Statistical Mechanics and its Applications 295, 53 - 57.

Baurmann, M., Gross, T., Feudel, U., 2007. Instabilities in spatially extended predator-prey systems: Spatio-temporal patterns in the neighborhood of Turing-Hopf bifurcations. Journal of Theoretical Biology 245, 220 - 229.

Berryman, A., Millstein, J., 1989. Are ecological systems chaotic - and if not, why not? Trends in Ecology and Evolution 4, $26-28$. 
Chakraborty, A., Singh, M., Lucy, D., Ridland, P., 1996. A numerical study of the formation of spatial patterns in twospotted spider mites. Mathematical and Computer Modelling 49, 1905-1919.

Colombo, A., Dercole, F., Rinaldi, S., 2008. Remarks on metacommunity synchronization with application to prey-predator systems. The American Naturalist 171, 430-442.

Cronberg, G., 1982. Changes in the phytoplankton of Lake Trummen induced by restoration. Hydrobiologia 86, 185-193.

Decima, M., Ohman, M.D., De Robertis, A., 2010. Body size dependence of euphausiid spatial patchiness. Limnology and Oceanography 55, 777-788.

Della Rossa, F., Fasani, S., Rinaldi, S., 2012. Potential Turing instability and application to plant-insect models. Mathematical and Computer Modelling $55,1562-1569$.

Doveri, F., Scheffer, M., Rinaldi, S., Muratori, S., Kuznetsov, Y., 1993. Seasonality and chaos in a plankton fish model. Theoretical Population Biology $43,159-183$.

Fasani, S., Rinaldi, S., 2011. Factors promoting or inhibiting Turing instability in spatially extended prey-predator systems. Ecological Modelling $222,3449-3452$.

Fasani, S., Rinaldi, S., 2012. Remarks on cannibalism and pattern formation in spatially extended prey-predator systems. Nonlinear Dynamics 67, $2543-2548$. 
Franks, P., 1997. Spatial patterns in dense algal blooms. Limnology and Oceanography 42, 1297-1305.

Gower, J., 1980. Phytoplankton patchiness indicates the fluctuation spectrum of mesoscale oceanic structure. Nature 288, 157 - 159.

Harris, G., 1986. Phytoplankton Ecology: Structure, Function and Fluctuation. Chapman and Hall, London.

Hastings, A., Hom, C., Ellner, S., Turchin, P., Godfray, H., 1993. Chaos in Ecology: Is mother Nature a strange attractor? Annual Review of Ecology and Systematics 24, 1-33.

Hillary, R., Bees, M., 2004a. Plankton lattices and the role of chaos in plankton patchiness. Phys. Rev. E 69, 031913.

Hillary, R., Bees, M., 2004b. Synchrony and chaos in patchy ecosystems. Bulletin of Mathematical Biology 66, 1909 - 1931.

Huang, Y., Diekmann, O., 2003. Interspecific influence on mobility and turing instability. Bullettin of Mathematical Biology 65, 143-156.

Leah, R., Moss, B., Forrest, D., 1980. The role of predation in causing major changes in the limnology of a hyper-eutrophic lake. Int. Revue ges. Hydrobiol. Hydrogr. 65, 223 - 247.

Levin, S., Segel, L., 1976. Hypothesis for origin of planktonic patchiness. Nature 259, 659 .

Levin, S.A., 1992. The problem of pattern and scale in ecology: the Robert H. MacArthur award lecture. Ecology 73, 1943-1967. 
Levitan, C., Kerfoot, C., De Mott, W., 1985. Ability of Daphnia to buffer trout lakes against periodic nutrient. Verh. Int. Verein. Limnol. 22, 30763082.

Li, Z., Gao, M., Hui, C., Han, X., Shi, H., 2005. Impact of predator pursuit and prey evasion on synchrony and spatial patterns in metapopulation. Ecological Modelling 185, 245-254.

Malchow, H., 1993. Spatio-temporal pattern formation in nonlinear nonequilibrium plankton dynamics. Proceedings of the Royal Society of London. Series B: Biological Sciences 251, 103-109.

Malchow, H., 1994. Nonequilibrium structures in plankton dynamics. Ecological Modelling 75-76, 123 - 134.

May, R., 1974. Biological populations with nonoverlapping generations: Stable points, stable cycles, and chaos. Science 186, 645-647.

Medvinsky, A., Petrovskii, S., Tikhonova, I., Malchow, H., Li, B.L., 2002. Spatiotemporal complexity of plankton and fish dynamics. SIAM Rev. 44, $311-370$

Murray, J.D., 1990. Mathematical Biology. Springer-Verlag, Berlin, German.

Okubo, A., 1992. Diffusion and Ecological Problems: Mathematical Models. Springer-Verlag, Berlin.

Pascual, M., Ascioti, F., Caswell, H., 1995. Intermittency in the plankton: A multifractal analysis of zooplankton biomass variability. J. Plankton Res. 17, 1209-1232. 
Powell, T., Okubo, A., 1994. Mutual interference between predators can give rise to Turing spatial patterns. Phil. Trans. R. Soc. Lond. B 343, 11-18.

Reinertsen, H., Olsen, Y., 1984. Effects of fish elimination on the phytoplankton community of a eutrophic lake. Verh. Int. Verein. Limnol. 22, 649-657.

Rinaldi, S., Solidoro, C., 1998. Chaos and peak-to-peak dynamics in a plankton-fish model. Theoretical Population Biology 54, 62 - 77 .

Rose, K., Swartzman, G., Kindig, A., Taub, F., 1975. Digestive activities of carp as major contributor to the nutrient loading of lakes. Verh. Int. Verein. Limnol. 19, $2461-2468$.

Rose, K., Swartzman, G., Kindig, A., Taub, F., 1988. Stepwise iterative calibration of a multi-species phytoplankton-zooplankton simulation model using laboratory data. Ecological Modelling 42, 1 - 32 .

Satnoianu, R., van den Driessche, P., 2005. Some remarks on matrix stability with application to Turing instability. Linear Algebra and its Applications $398,69-74$.

Satnoianu, R., Menzinger, M., Maini, P., 2000. Turing instabilities in general systems. Journal of Mathematical Biology 41, 493-512.

Scheffer, M., 1991a. Fish and nutrients interplay determines algal biomass: a minimal model. Oikos 62, $271-282$.

Scheffer, M., 1991b. Should we expect strange attractors behind plankton dynamics - and if so, should we bother? J. Plankton Res. 13, 1291-1305. 
Segel, L., Jackson, J., 1972. Dissipative structure: An explanation and an ecological example. Journal of Theoretical Biology 37, 545 - 559 .

Shanks, A., 1983. Surface slicks associated with tidally forced internal waves may transport pelagic larvae of benthic invertebrates and fishes shoreward. Mar. Ecol. Prog. Ser. 13, 311-315.

Shapiro, J., Wright, D., 1984. Lake restoration by biomanipulations, round Lake Minnesota - the first two years. Freshwater Biol. 14, 371-383.

Steele, J.(ed.)., 1978. Spatial Pattern in Plankton Communities. New York; Plenum.

Steele, J.H., Henderson, E.W., 1992. A simple model for plankton patchiness. J. Plankton Res. 14, 1397-1403.

Sugihara, G., May, R., 1990. Nonlinear forecasting as a way of distinguishing chaos from measurement error in time series. Nature 344, 734-41.

Sun, G., Zhang, G., Jin, Z., 2009. Predator cannibalism can give rise to regular spatial pattern in a predator-prey system. Nonlinear Dynamics 58, $75-84$.

Turing, A., 1952. The chemical basis of morphogenesis. Philosophical Transactions of the Royal Society of London. Series B, Biological Sciences 237, $37-72$.

Vilar, J., Solé, R., Rubí, J., 2003. On the origin of plankton patchiness. Physica A: Statistical Mechanics and its Applications 317, 239 - 246. 
505

Walters, C., Martell, S., 2004. Fisheries Ecology and Management. Princeton University Press, Princeton, NJ.

Wang, W., Liu, Q.X., Jin, Z., 2007. Spatiotemporal complexity of a ratiodependent predator-prey system. Phys. Rev. E 75, 051913.

Yodzis, P., 1994. Predator-prey theory and management of multispecies fisheries. Ecological Applications 4, 51-58.

Zhang, L., Wang, W., Xue, Y., 2009. Spatiotemporal complexity of a predator-prey system with constant harvest rate. Chaos, Solitons and Fractals 41, $38-46$. 